\title{
Surface properties of Rosetta's targets (21) Lutetia and (2867) Steins from ESO observations ${ }^{\star}$
}

\author{
J. M. Carvano ${ }^{1,2}$, M. A. Barucci ${ }^{2}$, M. Delbó ${ }^{3}$, S. Fornasier ${ }^{2,4}$, S. Lowry ${ }^{5,6}$, and A. Fitzsimmons ${ }^{5}$ \\ 1 Observatório Nacional (COAA), rua Gal. José Cristino 77, São Cristóvão, CEP20921-400 Rio de Janeiro RJ, Brazil \\ e-mail: carvano@on.br \\ ${ }^{2}$ LESIA - Observatoire de Paris, 5 Place Jules Janssen, 92195 Meudon Principal Cedex, France \\ 3 Laboratoire Cassiopée, Observatoire de la Cote d'Azur, BP 4229, 06304 Nice Cedex 04, France \\ ${ }^{4}$ Université de Paris 7 Denis Diderot, France \\ 5 Astrophysics Research Centre, Physics Building, Queen's University Belfast, Belfast BT7 1NN, UK \\ ${ }^{6}$ NASA Jet Propulsion Laboratory [MS 183-301], 4800 Oak Grove Drive, Pasadena, CA 91109, USA
}

Received 11 June 2007 / Accepted 8 October 2007

\begin{abstract}
Aims. The aim of this work is to constrain the size, composition and surface properties of asteroids (2867) Steins and (21) Lutetia, targets of the Rosetta mission. Rosetta is en route to rendezvous with comet 67P/Churyumov-Gerasimenko.

Methods. Thermal-Infrared N-band observations for Lutetia and Steins were obtained using, respectively, TIMMI2 on the ESO 3.6-m telescope at La Silla and VISIR at the UT3 VLT telescope on Cerro Paranal; visible light curves for Steins were obtained using NTT+SUSI2, while $R$-band photometry for Lutetia was obtained with the 2.0-m Faulkes Telescope North on Haleakala. For Steins, the NEATM model was used to constrain its visible geometric albedo and beaming parameter. A detailed thermophysical model was implemented and used to analyze our set of observations of Lutetia as well as previous reported measurements.

Results. The visible photometry of Steins was used along with data from the literature to yield a slope parameter of $G=0.32_{-0.11}^{+0.14}$. Problems during the observations led to the loss of measurements on two of the three $N$-band filters requested for Steins. Using the remaining data and the polarimetric albedo recently published, we were able to constrain the thermal beaming parameter as $\eta>1.2$, which is more similar to near-Earth asteroids and suggests either high thermal inertia or a very rough surface. For Lutetia, the best fit visible geometric albedo obtained with our model and the reported observation is $p_{v}=0.129$, significantly lower than that obtained if one applies the same model to previously reported measurements. The discrepancy cannot be explained solely by assuming inhomogeneities in the surface properties and we suggest that the most plausible explanation is the presence of one or more large craters on the northern hemisphere. For both sets of measurements, the implied single scattering albedo of Lutetia is compatible with laboratory measurements of carbonaceous chondrite meteorites.
\end{abstract}

Key words. minor planets, asteroids - infrared: solar system

\section{Introduction}

Following the postponement of the original launch date of the Rosetta spacecraft and its successful launch on March 2nd 2004, asteroids (21) Lutetia and (2867) Steins were chosen to be the targets of the two asteroid flybys to be performed by the Rosetta spacecraft on its way to rendezvous with comet 67P/Churyumov-Gerasimenko.

(2867) Steins is a small main belt asteroid for which present knowledge is still very limited. The first visible and near infrared spectra of Steins reveal a spectral behavior consistent with an E-type asteroid, although a determination of its albedo is required for a definitive classification (Barucci et al. 2005). They show a strong feature at about $0.5 \mu \mathrm{m}$, a weaker one at about $0.96 \mu \mathrm{m}$ and a flat and featureless behavior beyond $1 \mu \mathrm{m}$, all these features being similar to those of (64) Angelina, a representative member of the sub-type II of the E class. A recent determination of the geometric albedo through polarimetry

\footnotetext{
* Based on observations performed at the European Southern Observatory (ESO), proposals 076.C-0682(A), 076.C-0682(B) and DDT 276.C-5019. The Faulkes Telescope North is operated by the Las Cumbres Observatory.
}

yield $p_{v}=0.45 \pm 0.10$ (Fornasier et al. 2006), which is consistent with E-type asteroids.

(21) Lutetia is a large main belt asteroid which has been observed extensively from the ground and also by the infrared satellite IRAS. The IRAS albedo of $p_{V}=0.23$ (Tedesco et al. 2004) led Lutetia to be initially classified as an M-type asteroid, a classification that implies a composition analogous to iron meteorites. This however was at odds with the geometric albedo of $p_{v}=0.1$ derived through polarimetry (Zellner \& Gradie 1976). This and subsequent polarimetric observations led Lupishko \& Belskaya (1989) to argue against an M-type classification for Lutetia and Belskaya \& Lagerkvist (1996) to propose CV meteorites as the best analogs to the polarimetric properties of the asteroid. Spectroscopic observations have shown that its infrared spectrum is unusually flat compared to other M-type asteroids and that it is similar to carbonaceous chondrite spectra which characterize the C-type asteroids (Birlan et al. 2004). The discovery of the $3 \mu \mathrm{m}$ absorption feature diagnostic of water of hydration (Rivkin et al. 2000) and of the possible presence of features at 0.44 and $0.67 \mu \mathrm{m}$ probably associated to hydrated silicates (Lazzarin et al. 2004) all favor a C-type classification for Lutetia. Recently Birlan et al. (2006) and Nedelcu et al. (2007) 
suggested that the NIR spectra of Lutetia is best matched by CV meteorites.

In this work we present new thermal-infrared and visible observations of Lutetia and Steins obtained with ESO telescopes and with the Faulkes telescope at Haleakala, Maui. Section 2 describes the observations and data reduction procedures; Sect. 3 describes the analysis of the visible observations for both asteroids while Sects. 4 and 5 deal with the interpretation of the thermal-infrared observations for Lutetia and Steins, respectively. The main results are summarized in Sect. 6.

\section{Observations}

\subsection{Thermal-infrared observations}

The thermal-infrared observations of Steins were performed with VISIR at the ESO $8.2 \mathrm{~m}$ Very Large Telescope UT3 (Lagage et al. 2004), while Lutetia was observed with TIMMI2 at the $3.6 \mathrm{~m}$ at La Silla (Käufl et al. 2003). Both asteroids were observed in service mode. Our observations included mediuminfrared photometric standard stars from the database of Cohen et al. (1999). For Steins, photometry in the PAH-1, SIV, and PAH-2 filters (central wavelength 8.58, 10.48 and $12.13 \mu \mathrm{m}$, respectively) was requested, as well as observations of the flux standard star HD 209688 in the same filters. The data in filter PAH-1 were acquired on 07 Oct., 2005, while the observations in the remaining filters were made on 04 Nov., 2005. Unfortunately, due to problems with the differential tracking during the November observation, the Steins' data in the SIV and PAH-2 filters turned out to be unusable, leaving us only with data in the PAH-1 filter. For Lutetia, observations in the N1, N10.4-OCLI and N12.9-OCLI filters were requested (central wavelength $8.7,10.49$ and $12.35 \mu \mathrm{m}$, respectively), along with a low dispersion $\mathrm{N}$ band spectrum. All data were acquired on 06 Jan., 2006 using HD87837 as a flux standard star. Table 1 lists the details of all thermal-infrared observations.

For both TIMMI2 and VISIR observations the initial steps of data reduction were handled by the respective pipelines. The observed flux of the photometric observations were obtained using aperture photometry, with the size of the aperture being determined using the photometric-grow curve. The fluxes were then corrected for atmospheric extinction and calibrated to physical units using the extinction and zero-point coefficients determined from the flux standard stars. For Steins, we obtained a flux at the PAH- 1 filter of $f_{8.58}=7.31 \times 10^{-16} \mathrm{~W} / \mathrm{m}^{2} / \mu \mathrm{m}$, while Lutetia the derived fluxes are $f_{8.7}=9.32 \times 10^{-14} \mathrm{~W} / \mathrm{m}^{2} / \mu \mathrm{m}, f_{10.35}=$ $1.24 \times 10^{-13} \mathrm{~W} / \mathrm{m}^{2} / \mu \mathrm{m}$ and $f_{12.35}=1.63 \times 10^{-13} \mathrm{~W} / \mathrm{m}^{2} / \mu \mathrm{m}$. The spectra of Lutetia and of HD 87837 were extracted in the standard fashion and calibrated in wavelength using the position of the $9.5 \mu \mathrm{m}$ telluric ozone feature line as reference, along with the wavelength calibration table of TIMMI2. The flux-calibrated spectrum of Lutetia was then obtained by dividing it by the observed spectrum of HD 87837 and then multiplying the result by the flux-calibrated spectrum of the star. To correct for slit losses we convolved the thermal-infrared spectrum with the band pass of the N10.4-OCLI filter and used the the calibrated flux at this filter to derive a scaling factor. Figure 1 shows the flux-calibrated spectrum and thermal-infrared photometry of Lutetia.

\subsection{Visible light observations}

Light curves of Steins in the $V$ band were obtained using SUSI2 on the ESO NTT in service mode on 21 Nov., 2005 and 28 Nov., 2005, comprising 3 and $2 \mathrm{~h}$ of observations
Table 1. Details of the thermal-infrared observations.

\begin{tabular}{ccccc}
\hline \hline Instument & Object & Time (UT) & Filter & Airmass \\
\hline VISIR & Steins & 07 Oct., 2005 00:32 & PAH-1 & 1.035 \\
VISIR & HD 209688 & 07 Oct., 2005 00:20 & PAH-1 & 1.088 \\
VISIR & HD 209688 & 07 Oct., 2005 01:58 & PAH-1 & 1.036 \\
TIMMI2 & Lutetia & 10 Jan., 2006 07:44 & N1 & 1.366 \\
TIMMI2 & Lutetia & 10 Jan., 2006 07:57 & N10.4 & 1.359 \\
TIMMI2 & Lutetia & 10 Jan., 2006 08:05 & N12.9 & 1.357 \\
TIMMI2 & HD 87837 & 10 Jan., 2006 09:14 & N1 & 1.414 \\
TIMMI2 & HD 87837 & 10 Jan., 2006 09:17 & N10.4 & 1.425 \\
TIMMI2 & HD 87837 & 10 Jan., 2006 09:20 & N12.9 & 1.433 \\
TIMMI2 & Lutetia & 10 Jan., 2006 08:57 & N band & 1.358 \\
TIMMI2 & HD 87837 & 10 Jan., 2006 09:09 & N band & 1.388 \\
\hline
\end{tabular}

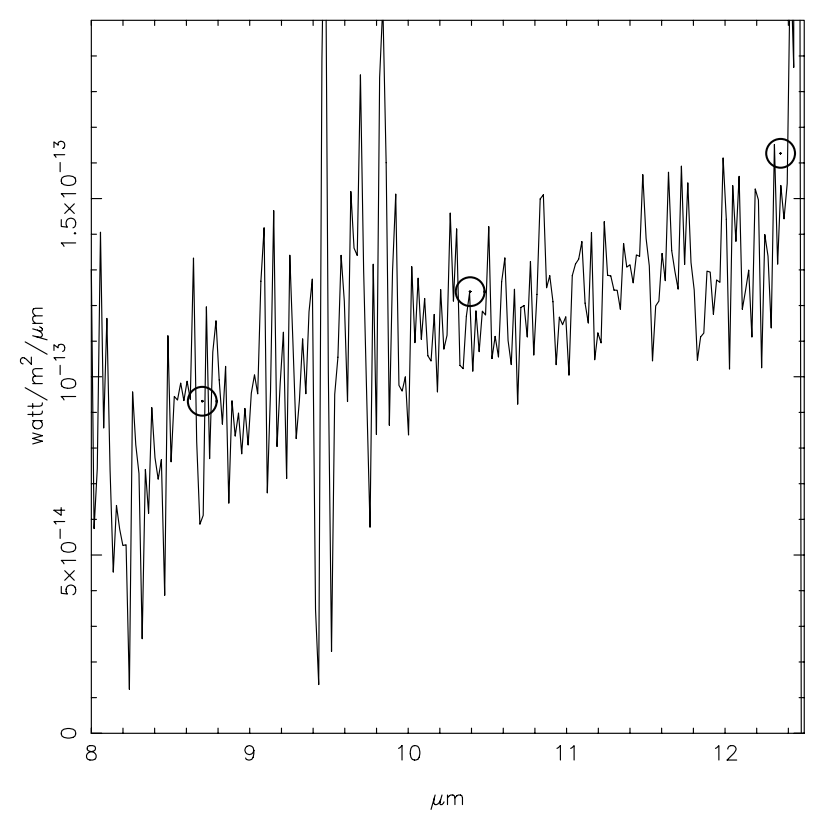

Fig. 1. Calibrated thermal-infrared flux of 21 Lutetia.

respectively. Light curves of Lutetia in the $V$ and $R$ bands were obtained using the 2.0-m Faulkes Telescope North, situated on the Hawaiian island of Maui, on 11 Dec., 2005 (1.5 h) and 24 Dec., 2005 (2 h).

For both datasets the data reduction was performed in the standard fashion, with bias and flat field corrections followed by aperture photometry on the asteroids, field stars and standard stars, with extinction coefficients calculated from the field stars and photometric zero points given by the standard stars. For Lutetia, the second night was affected by poor atmospheric conditions and no suitable calibration was possible.

\section{Analysis of the optical observations}

Our main goal here is to determine the absolute magnitude $H$ at the instants of the thermal-infrared observations.

For Steins, a reasonable concern is the lack of a reliable value for the slope parameter $G$. Recently, using their own observations in the $R$ band made at a phase angle of $17.07^{\circ}$ along with observations at a phase angle of $11.07^{\circ}$ by Hicks et al. (2004), Weissman et al. (2007) derived for Steins a slope parameter of $G_{R}=0.46_{-0.20}^{+0.32}$. Here we recalculate $G$ including our observations and the data of Küppers et al. (2007), who obtained photometry of Steins with the OSIRIS camera on board the Rosetta spacecraft. The reduced magnitudes (i.e., at $1 \mathrm{AU}$ 


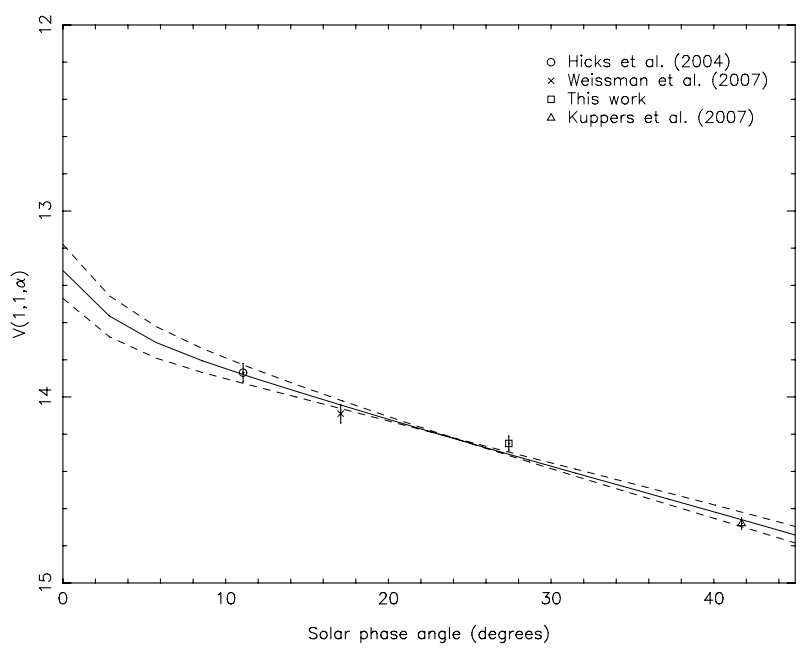

Fig. 2. Best fit HG parameters for (2867) Steins.

from the Sun and from the observer) in the $R$ band from Hicks et al. (2004) and from Weissman et al. (2007) $(R=13.36 \pm 0.02$ and $R=13.51$, respectively) were converted to the $V$ band using the $(V-R)$ colors derived in each work $((V-R)=0.51 \pm 0.02$ and $(V-R)=0.58 \pm 0.02)$. Küppers et al. (2007) observed Steins at a phase angle of $41.7^{\circ}$ using a clear filter and converted their results to the $V$ band, obtaining an average reduced magnitude of $V=14.68 \pm 0.03$. Here we use magnitudes observed during our first night, when Steins was at a phase angle of $27.4^{\circ}$. On this night we do not have full light curve sampling but rather a maximum and a minimum, therefore the average reduced magnitude is calculated as the mean of the magnitude at the light curve extrema, yielding $V=14.25 \pm 0.04$. Using the equation $V(\alpha)=H-2.5 \log \left[(1-G) \Phi_{1}(\alpha)+G \Phi_{2}(\alpha)\right]$ (see Bowell et al. 1989 , for the definition of $\Phi_{1}$ and $\Phi_{2}$ ) we fitted for $H$ and $G$ using a simplex fitting algorithm, using the nominal uncertainties to weight the measurements. To estimate meaningful uncertainties to the derived values of $H$ and $G$ we repeated the fitting procedure 10000 times with magnitudes values drawn randomly within the $1 \sigma$ interval of each measurement, obtaining $G=0.32_{-0.11}^{+0.14}$ and $H=13.32_{-0.14}^{+0.15}$ for Steins (Fig. 2).

In order to make a composite light curve from our observations we initially considered the rotational period of $6.052 \pm$ $0.007 \mathrm{~h}$ (Küppers et al. (2007) and then varied it slightly until the minima overlapped. The best agreement between the two nights was obtained with a period of $6.0455 \mathrm{~h}$, consistent with the value of Küppers et al. (2007) and of the period of $6.048 \pm 0.007 \mathrm{~h}$ derived by Weissman et al. (2007). The resulting light curve shows two maxima and a minimum, with an amplitude of $0.24 \pm 0.07$. Figure 3 a shows the composite light curve ( $H$ versus rotational phase) for Steins.

For Lutetia, $H$ was calculated using the $G$ value of 0.11 from the IRAS minor Planet Survey v6.0 (Tedesco et al. 2004). Due to the limited extent of the observed light curve we used the rotation period, pole determination and shape model of Lutetia from Torppa et al. (2003) to generate a synthetic light curve for Lutetia, that was matched with the observed light curve in order to extrapolate the rotational phase to the instants of the thermalinfrared observations. The agreement between the synthetic and observed lightcurves is excellent (Fig. 3b).

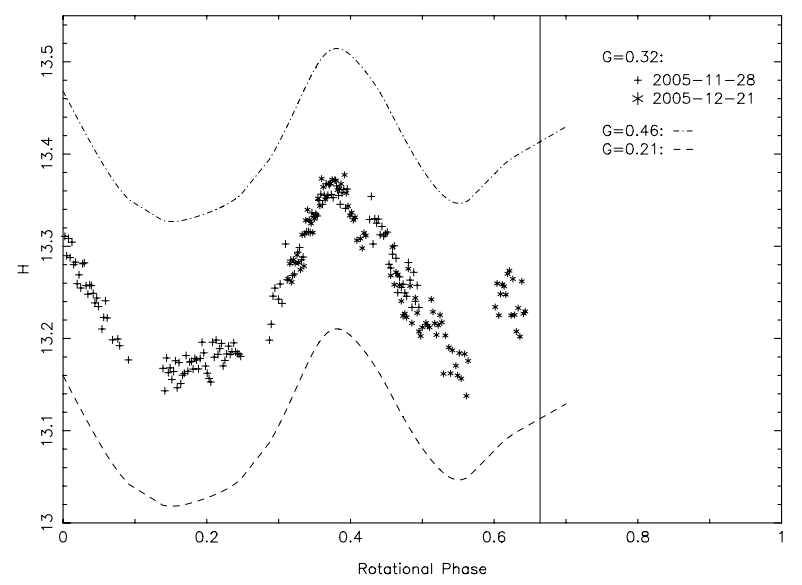

(a)

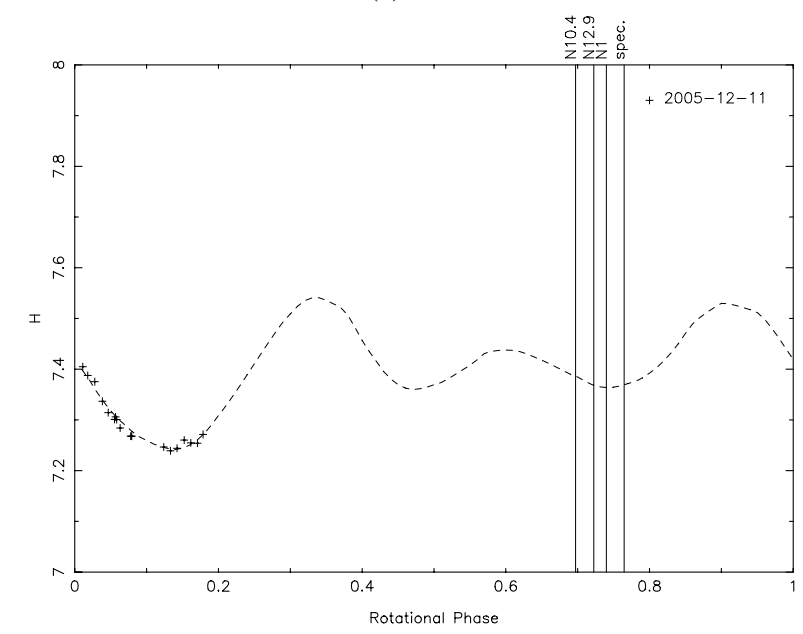

(b)

Fig. 3. a) Absolute magnitude versus rotational phase for Steins. The solid line marks the rotational phase of the VISIR measurement. b) Absolute magnitude versus rotational phase for Lutetia. The synthetic light curve was made considering pole $\beta=+3^{\circ}, \lambda=39^{\circ}$ and a rotation period $=8.165455 \mathrm{~h}$ (Torppa et al. 2003).

\section{Lutetia}

\subsection{Model fitting}

The high quality thermal-infrared spectra obtained for Lutetia and the existence of a shape model and pole solutions for this asteroid makes it a good candidate for the use of a thermophysical model, where the thermal emission is modeled taking into account the vertical thermal conduction into the regolith layer and the surface rugosity (Spencer 1990; Lagerros 1996). A concern in the implementation of the thermophysical model was to take into full consideration the non-spherical shape of Lutetia. Because of that we decided not to explicitly consider the visible geometric albedo $p_{v}$ as a free parameter of the model, using instead the spherical radius $r_{s}$ and the volumetric single scattering albedo (see Appendix A for a definition of $r_{s}$ and its relations to $p_{v}$ and the effective radius $r_{d}$ ). Our thermophysical model has four free parameters: the single scattering volumetric albedo $(w)$ of the material on the surface, the surface thermal parameter $(\Theta)$, the fraction of the surface covered with spherical craters $(f)$ and the relative depth of the craters $(h)$. The model is described in more detail in Appendix B.

In the fitting procedure we adopted the shape model and the first pole solution of Torppa et al. (2003), with ecliptic 


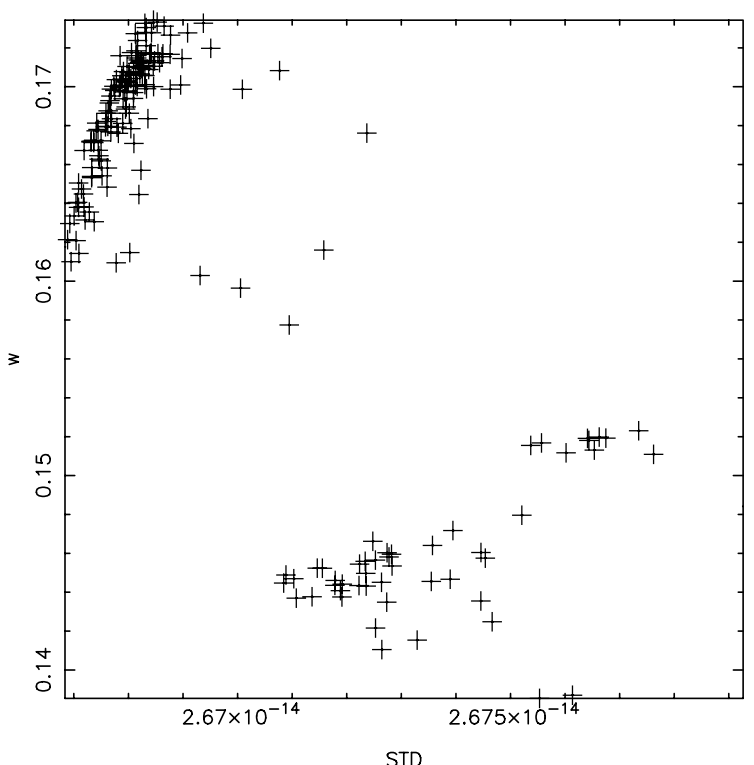

(a)

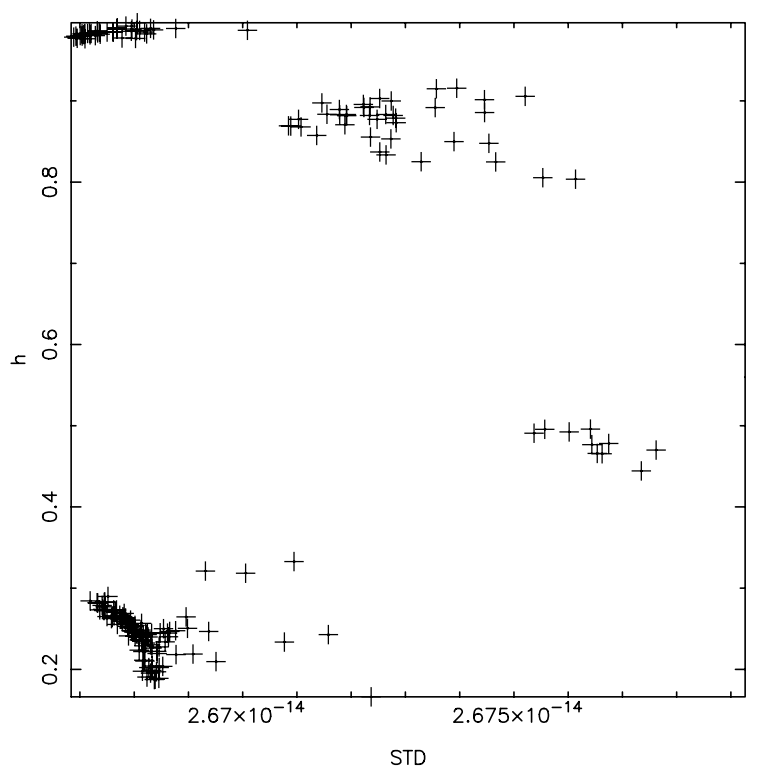

(c)

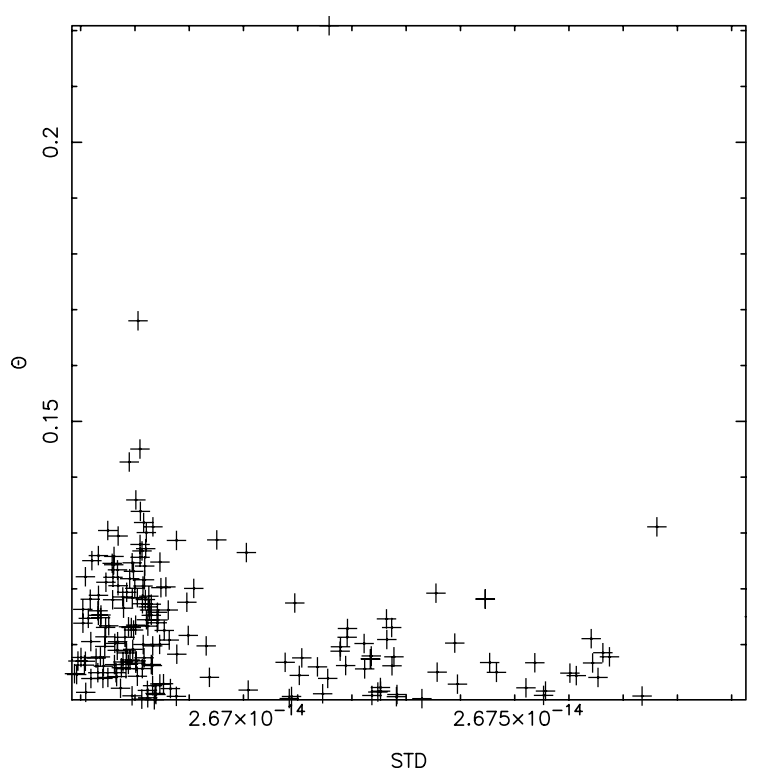

(b)

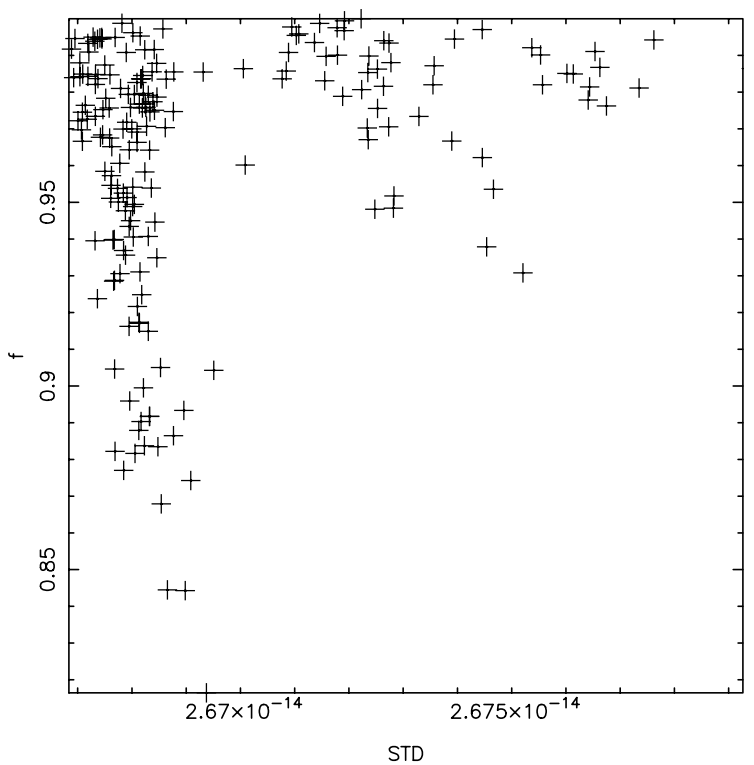

(d)

Fig. 4. Fitted solutions of the thermophysical model parameters for Lutetia: a) single scattering albedo, b) thermal parameter, c) crater depth and d) faction of crater coverage.

coordinates $\lambda=39^{\circ}$ and $\beta=3^{\circ}$. The phase function parameters were chosen in order to produce an integral phase function consistent with the slope parameter $G=0.11$ that is assumed for Lutetia (see Sect. 4.2.2). The absolute magnitude and subearth longitude of Lutetia at the moment of the thermal-infrared observations were determined from the fit of the synthetic light curve to the observed one.

In order to obtain a more complete mapping of the parameter space and to allow for the existence of multiple solutions, the fitting was made using a genetic algorithm set to perform deterministic crowding. Genetic algorithms are general purpose, parallel search procedures that are based upon genetic and evolutionary principles (Goldberg 1989; Holland 1992; Mahfoud 1995). The genetic algorithm used in this work was based on the GAlib implementation (http://lancet.mit.edu/ga). With a careful choice of the mating and mutation strategies it is possible to set the algorithm to operate in the deterministic crowding mode, where the multiple sets of fit solutions found during the evolution are preserved, allowing the simultaneous determination of most (hopefully all) solutions of a given problem (Mahfoud 1995). We used a population of 200 individuals and let the evolution proceed for 150 generations. The distribution of the fitted parameters versus the standard deviation of the fit are shown in Fig. 4. The best solutions exhibit single scattering albedo around $w=0.165$ (with another cluster of solutions around $w=0.145$, but of slightly lesser quality), thermal parameter $\Theta<0.13$, fractional crater coverage $f>0.90$ and a bimodal distribution of relative crater depth, with good solutions around $h=0.22$ and $h=0.97$. We then use the model parameters to calculate a set of parameters that are more usual or more physically relevant: the visible geometric albedo $p_{v}$, the surface thermal inertia $\Gamma$ and the RMS slope. The thermal inertia is related to the surface 
microporosity and conductivity, while the RMS slope is a measure of the macroscopic roughness of the surface (Spencer 1990; Lagerros 1996). The geometric albedo on the other hand is the parameter that is often quoted in the literature and it is calculated here to allow direct comparison with previous works. The median values of the calculated parameter (considering only the fitted solutions) are $p_{v}=0.129_{-0.030}^{+0.003}, \Gamma=5.2_{-0.3}^{+0.9} \mathrm{~J} \mathrm{~m}^{-2} \mathrm{~K}^{-1} \mathrm{~s}^{-1 / 2}$ and $27_{-2}^{+3}$ or $48_{-2}^{+1}$ degrees for the RMS slope. The uncertainties quoted refer only to the scatter on the fitted parameters. The actual uncertainties should be somewhat higher, since they must include the uncertainties in $G$, in the pole determination and in the shape model. Figure 5 shows the scatter plots of these parameters.

\subsection{Discussion}

\subsubsection{The visible geometric albedo conundrum}

One of the motivations of this work was the disagreement between the geometric albedo determinations of Lutetia in the literature (2). Prior to 2007, the visible geometric albedo determined through radiometry yielded $p_{v}=0.2$, polarimetry favors $p_{v}=0.1$ and the radar determination could be consistent with both, within the measurement uncertainties. The more recent work seems only to add to the confusion: new polarimetry by Gil-Hutton (2007) yield $p_{v}=0.1$ if one uses the $P_{M I N}$ calibration, but $p_{v}=0.2$, if the $h$ calibration is used.

Considering only the thermal-infrared measurements, the discrepancies may be explained as problems with the calibration of some of the sets of observations, as caused by problems with the models used to interpret the data or as real variations in some of the assumed properties of the surface. To adress the possibility of calibration problems on our measurements, an independent reduction was performed on our data and the results were found to be consistent with those presented here. To test if some particularities in our implementation of the thermophysical model could be to blame, we applied our model to the observations reported by Mueller et al. (2006) obtaining $r_{s}=57 \mathrm{~km}$, which translates to a visible geometric albedo of $p_{v}=0.235$ and an effective radius of $r_{d}=47 \mathrm{~km}$, consistent with the values reported by the authors. Variations in the observation geometry only can not explain this difference, since they are fully accounted for in our model.

Considering the sub earth-latitudes involved in the thermalinfrared measurements (Table 2) a north-south asymmetry seems at first a possible explanation. The measurements presented in this work were made at $l_{\oplus} \approx 26^{\circ}$, while the measurements for $p_{v}=0.2$ were made at $l_{\oplus}=-75^{\circ}$ and $l_{\oplus}=-3^{\circ}$, therefore the albedo dichotomy might be explained if one assumes that a portion of the northern hemisphere has different albedo, thermal inertia and/or roughness than the rest of the asteroid. However, for an asteroid with a fixed spherical radius, even large variations in any of the parameters are not capable of producing changes in the thermal-infrared flux that could explain the variations observed.

If we rule out real albedo variations and calibration issues, the only explanation for our model to yield two different results at two different instants is that one or more of the hypothesis that go into the model are violated. In particular, we assume a convex shape for Lutetia and use a shape model that was obtained with the same assumption. One possible way of explaining the visible geometric albedo dichotomy is to suppose the existence of a large crater in the northern hemisphere: the self-heating effect of the radiation reflected and emitted by the crater walls would

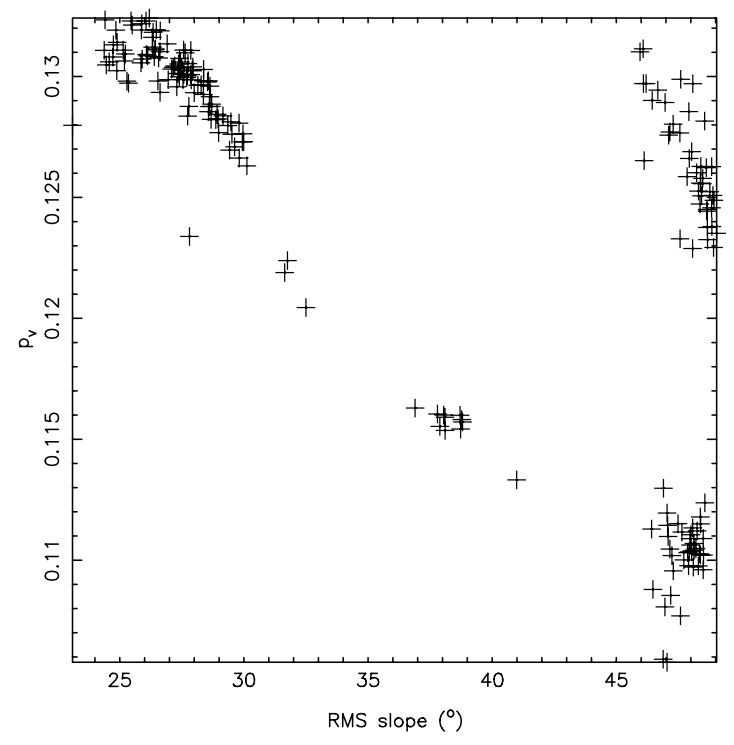

(a)

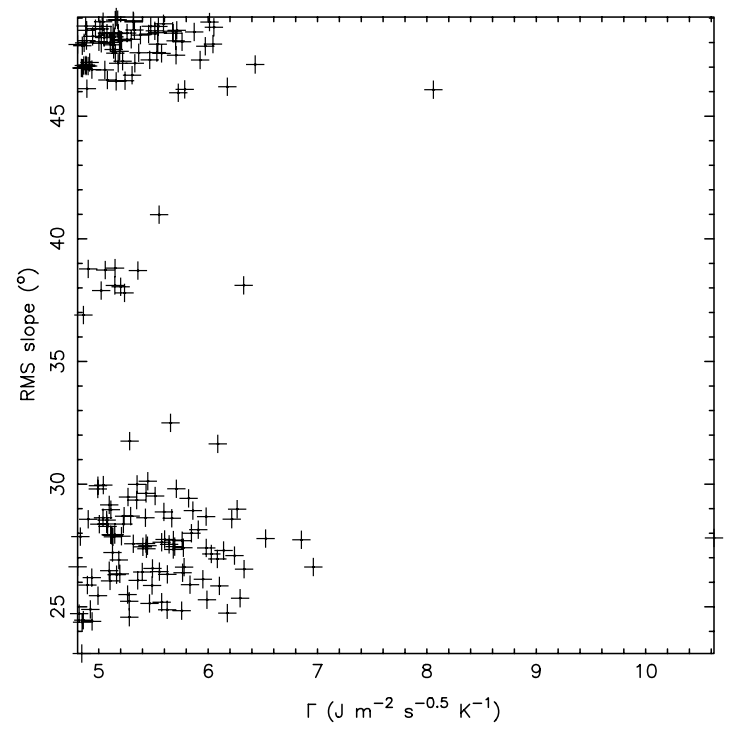

(b)

Fig. 5. Distribution of the fitted solutions for parameters derived from the thermophysical model for Lutetia: a): RMS slope versus visible geometric albedo; b) thermal inertia versus RMS slope.

enhance the thermal-infrared flux observed at northern sub-earth latitudes, and that would be adjusted by our model by assigning a larger radius to the asteroid at those particular viewing geometries. If that is the case the model used in this work is not adequate, and the derived values for the model parameter should be regarded with caution. For the sake of completeness the results for single scattering albedo, thermal inertia and RMS slope are discussed in the remainder of this section.

\subsubsection{Compositional constraints}

It is common in the literature to use the visible geometric albedo as an indicator of composition. However, the volumetric single scattering albedo of the surface is actually the relevant parameter to be considered, since the visible geometric albedo (not surprisingly) is also related to shape and viewing geometry. Even a spherical body will have a visible geometric albedo lower than 
Table 2. Geometric albedo determinations for (21) Lutetia.

\begin{tabular}{cccccc}
\hline \hline Author & Obs. & $R(\mathrm{AU})$ & $l_{\oplus}$ & $l_{\odot}$ & $p_{v}$ \\
\hline Tedesco et al. (1992) & Thermal & 2.825 & -3 & 17 & $0.221 \pm 0.020$ \\
Zellner \& Gradie (1976) & Pol. & - & - & - & 0.1 \\
Magri et al. (1999) & Radar & - & - & & $0.151 \pm 0.045$ \\
Muller et al. (2006) & Thermal & 2.065 & -75 & -48 & $0.225 \pm 0.045$ \\
Gil-Hutton (2007) & Pol. $\left(P_{\min }\right)$ & - & & & $0.11 \pm 0.01$ \\
Gil-Hutton (2007) & Pol. $(h)$ & - & & & $0.20 \pm 0.04$ \\
Present work & Thermal & 2.823 & +25 & +10 & $0.129_{-0.030}^{+0.003}$ \\
\hline
\end{tabular}

the volumetric single scattering albedo of the particles on its surface (i.e. Hapke 1993).

The model used in this work allows a direct estimation of the single scattering albedo accounting for the geometry of the observation and the shape of the body. However, the single scattering albedo derived in this way is sensitive to the volumetric phase function of the material, which cannot be properly constrained from disk-integrated observations. Instead, the brightness variation of disk-integrated photometry of asteroids is usually modeled through a semi-empirical integral phase function that is a function of the slope parameter $G$ (Bowell et al. 1989).

In order to estimate the phase parameters $b$ and $c$ of the double-lobed Heyney-Greenstein function used in this work in a fashion that is at least consistent with the inferred phase behavior of Lutetia, we considered a sphere with bi-directional reflectance described by a Hapke-like function (see Appendix B) and looked for $(b, c)$ that more closely reproduced a Bowell phase function with $G=0.11$. We found that for single scattering albedo in the $0.01-0.4$ range the Lutetia's assumed integral phase function $(G=0.11)$ is best matched with $b \in[0.36,0.39]$ and $c \in$ $[0.7,0.79]$. We therefore adopted $b=0.375$ and $c=0.75$ as input for the thermal model. With these values, the model infrared flux is best matched to our observations with $w=0.169_{-0.009}^{+0.002}$. However, for the observations of Mueller et al. (2006), the model yields $w=0.35$ for the same input values.

A single scattering albedo of $w \approx 0.17$ is compatible with the value of $w=0.15 \pm 0.02$ (Piironen et al. 1998) of the C2 meteorite Kivesvaara, while $w \approx 0.3$ matches the measured albedo of the CV3 meteorite Allende (Kamei \& Nakamura 2002). Therefore, both values agree with compositional inferences from polarimetry (Belskaya \& Lagerkvist 1996) and spectroscopy in the NIR (Birlan et al. 2006; Nedelcu et al. 2007) and in the thermal infrared (Barucci et al., in preparation), which suggests that the composition of Lutetia is compatible with carbonaceous chondrite meteorites, in particular the CV chondrites.

\subsubsection{Thermal inertia and RMS slope}

The derived value for the thermal inertia is 10 times smaller than the thermal inertia of the Moon and consistent with derived values for large main-belt asteroids (Spencer \& Lebofsky 1986; Muller \& Lagerros 1998). Such low thermal inertia is generally interpreted as evidence for a well-developed regolith layer with a low thermal conductivity, which might be indicative of high surface microporosity. The thermal inertia derived in this work is also 10 times smaller than the reported best fit value of Mueller et al. (2006), who applied a thermophysical model to MIRSI thermal photometry, though the authors are careful to state that their dataset could not put stringent constraints on the thermal inertia and it could be fit assuming $\Gamma=0$. Our determinations of the RMS slope are the first in the literature for Lutetia. We note that the lower value is very close to the radar RMS slope

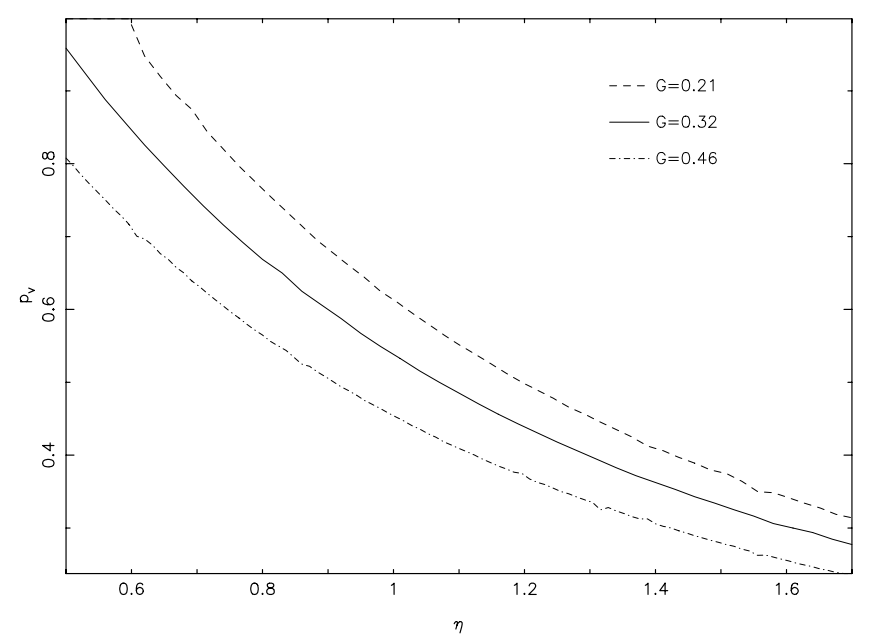

Fig. 6. NEATM fits to Steins.

calculated by Ostro et al. (1985) for (2) Pallas $\left(27^{\circ}\right)$. In principle, it would be possible to estimate the radar RMS slope from the observations reported by Magri et al. (1999), but the calculations involved are beyond the scope of this work.

\section{Steins}

Steins has too few data points for a thermophysical model. With a two parameter model like NEATM (Delbó \& Haris 2002), where one consider both the geometric albedo $\left(p_{v}\right)$ and the beaming parameter $(\eta)$ as free parameters, one can only constrain the possible solutions as curves in the $p_{v} \times \eta$ space. The exact location of these curves are rather sensitive to the adopted value of the slope parameter. Figure 6 shows the best fit $p_{v} \times \eta$ curves for Steins for the best determination and lower and upper values of $G$ derived in this work. A value of $\eta=0.756$ typical of Main-Belt asteroids would lead to unrealistic high values of the geometric albedo (0.6-0.9). If on the other hand we adopt the geometric albedo of $p_{v}=0.45$ determined through polarimetry (Fornasier et al. 2006) we obtain for the beaming parameter $\eta=1.2_{-0.2}^{+0.25}$. This value is more similar to near-Earth asteroids, and is suggestive of either a high thermal inertia or a highly rough surface.

\section{Conclusions}

In this work we present new thermal-infrared and visible observations of Lutetia and Steins. For Steins we were able to refine the value of the slope parameter and to put loose constraints on the thermal inertia and/or rugosity of its surface.

For Lutetia the main result presented here is the suggestion that the discrepancies in the thermal-infrared observations could 
be explained if one assumes the presence of large craters in the northern hemisphere. In order to investigate this possibility further it will be necessary to apply models that are able to deal with concavities to the whole set of visible and thermal-infrared observations of Lutetia. The other important result presented here is that the single scattering albedo of Lutetia derived from either sets of thermal-infrared measurements are compatible with values obtained in the lab for carbonatious chondrites, which is fully consistent with the current interpretation of spectroscopic data.

Acknowledgements. J. Carvano was supported by fellowships from the European Space Agency (ESA) and Conselho Nacional de Pesquisa Científica (CNPq). The work of M. Delbo was supported by the European Space Agency (ESA). S.C.L. acknowledges support from the Leverhulme Trust.

\section{Appendix A: Definition of the spherical radius}

We define the spherical radius as the radius of the sphere with the same area as the body: $4 \pi r_{s}^{2}=\int \mathrm{d} A$. Since the dimensions in the shape models are given in arbitrary units we define an area element $\mathrm{d} a=r_{\text {eff }}^{-2} \mathrm{~d} A$, and normalize the discrete area element in the shape model so that $\sum \delta a=4 \pi$. In order to link the so defined spherical radius to the more usual effective radius $r_{d}$ we must revise the formal definition of $p_{v}$. The visible geometric albedo $p_{v}$ is defined (i.e., Hapke 1993) as the ratio of the brightness of a body at zero solar phase angle to the brightness of a perfect Lambertian disk of the same radius and at the same distance as the body, but illuminated and observed perpendicularly. This of course only makes sense for a spherical body, but we can generalize this definition to an arbitrary shape by requiring that the area of the Lambertian disk be equal to the projected visible area of the body: $\pi r_{d}^{2}=r_{s}^{2} \int_{\text {Visible }} \mu_{e} \mathrm{~d} a$, where $\mu_{e}$ is the cosine of the angle between the direction of the observer and the normal of the surface element. For an spherical body $r_{s}=r_{d}$, preserving the original definition, but for a non-spherical convex body we will in general have $r_{s}>r_{d}$. The visible geometric albedo can then be calculated using the relation $r_{d}=\frac{1329}{2 \sqrt{p_{v}}} 10^{-0.2 H}$ (i.e. Bowell et al. 1989). Note that for a non-spherical body $r_{d}$ and $p_{v}$ will vary with the observing geometry.

\section{Appendix B: Implementation of the Thermophysical Model}

The thermophysical model used in this work combines several features of other previous modeling efforts, and an important concern was that its implementation allows that all free parameters can be simultaneously adjusted through fitting algorithms.

The model considers convex bodies described by a set of triangular facets, where the normalized temperature (i.e. the facet temperature expressed in units of the sub-solar temperature) of each body facet is given by the transport equation:

$\frac{\partial u}{\partial t}=\frac{\partial^{2} u}{\partial z^{2}}$

with the boundary condition at the surface given by

$u^{4}=\left.\Theta \frac{\partial u}{\partial z}\right|_{z=0}+\mu_{0}$.

In these equations $u$ is the normalized temperature, $\mu_{0}$ is the cosine of the solar incidence angle to the facet's normal and $\Theta$ is the thermal parameter,

$\Theta=\frac{\Gamma \sqrt{\omega}}{\epsilon_{h} \sigma T_{s s}^{3}}$ where $\Gamma$ is the thermal inertia, $\omega$ is the body's angular rotation rate $\epsilon_{h}$ is the hemispherical emissivity and $T_{s s}$ is the sub-solar temperature (see Lagerros 1996 for a deeper discussion). In this implementation, the normalized temperature was calculated as a function of the thermal parameter, sub-solar latitude, longitude and latitude of the normal of the facet. The resulting temperatures were stored in a table, which was interpolated during the fitting. To model the rugosity of the surface we followed Spencer (1990) and consider that a fraction $f$ of each facet is covered by craters that are sections of spherical craters of unity radius, described by its height $h$ (a hemispherical crater would have $h=1$ ). Each crater was divided in a number of tiles with equal area and the normalized temperature of each tile was calculated considering the vertical thermal conduction and the visible and thermal radiation scattered into it by the other tiles, correcting for shadowing; only single scatter by isotropic particles was considered. In practice, we considered 24 tiles per crater and calculated the normalized temperature for each as a function of their thermal parameters, sub-solar latitudes, longitudes and latitudes of the crater normal, crater height and single scattering albedo. The resulting table was interpolated during the fitting to yield the normalized temperature of each crater. The model thermal-infrared flux of the body was then calculated as

$$
\begin{aligned}
F_{\lambda}= & \frac{r_{s}^{2}}{\Delta^{2}}\left\{\sum_{i}\left[(1-f) \mu_{e_{i}} \epsilon_{d_{i}} B_{\lambda}\left(T_{s s} u_{i}\right)\right] \delta a_{i}\right. \\
& \left.+\sum_{i}\left[\frac{f}{a_{\mathrm{rim}}(h)} \sum_{j}\left[\mu_{e_{i j}} \epsilon_{d_{j i}} B_{\lambda}\left(T_{s s} u_{i j}\right) a_{\mathrm{tile}}\right]\right] \delta a_{i}\right\}
\end{aligned}
$$

where $r_{s}$ is the spherical radius of the body (see Appendix B), $\Delta$ is the distance to the observer, $\mu_{e}$ is the cosine of the angle between the direction of the observer and the normal of the element in consideration, $\epsilon_{d}$ is the directional emissivity of the material $=T_{S S}$ is the subsolar temperature of the body, $B_{\lambda}$ is the blackbody emission, $a_{\text {rim }}$ is the area of the crater rim, $a_{\text {tile }}$ the area of a crater tile and $\delta a$ is the solid angle of each facet (see Appendix B). The sub-solar temperature is given by (i.e. Spencer 1990)

$T_{s s}=\left\{\frac{(1-A) S_{\odot}}{\epsilon_{h} \sigma R^{2}}\right\}^{1 / 4}$

where $A$ is the bond albedo, $S_{\odot}$ is the solar constant and $R$ is the heliocentric distance of the body. The bond albedo and the hemispheric and directional emissivities can be expressed as functions of the volumetric single scattering albedo $w$ (see Hapke 1993). Finally, $r_{s}$ is related to $w$ through

$$
r_{s}=\frac{1329}{2 \sqrt{\sum_{i} B D\left(w, \mu_{0}, \mu_{e}, 0\right) \mu_{e} \delta a}} 10^{-0.2 H}
$$

where $B D$ is the bi-directional reflectance. Here we use Hapke's function

$$
B D\left(w, \mu_{0}, \mu_{e}, 0\right)=\frac{w}{4 \pi} \frac{\mu_{0}}{\mu_{0}+\mu_{e}}\left[p(0)+H\left(\mu_{0}\right) H\left(\mu_{e}\right)-1\right]
$$

where $p$ is the volumetric phase function and $H$ is the Chandrasekar function (see Hapke 1993). For the phase function we used a double-lobed Heyney-Greenstein function

$$
p(g)=\frac{1+c}{2} \frac{1-b^{2}}{\left(1-2 b \cos g+b^{2}\right)^{3 / 2}}+\frac{1-c}{2} \frac{1-b^{2}}{\left(1+2 b \cos g+b^{2}\right)^{3 / 2}} .
$$


Therefore, the model thermal-infrared flux depends on $w$ through the sub-solar temperature, the scattering inside the craters, the directional emissivity and $r_{s}$. In practice, however, the dependence on $r_{s}$ is much stronger, to the point that $r_{s}$ can effectively be considered an independent parameter, with $w$ being set by its relation to $r_{s}$. Indeed, the fitted value of $r_{s}$ is only slightly affected by the choice of the phase function parameters $b$ and $c$, while these parameters have a strong influence on the final value of $w$.

\section{References}

Barucci, M. A., Fulchignoni, M., Fornasier, S., et al. 2005, A\&A 430, 313 Belskaya, I. N., \& Lagerkvist, C.-I. 1996, Planet. Space. Sci. 44, 783 Birlan, M., Barucci, M. A., Vernazza, P., et al. 2004. New Astron., 9, 343 Birlan, M., Vernazza, P., Fulchignoni, M., et al. 2006, A\&A, 454, 677

Bowell, E., Hapke, B., Domingue, D., et al. 1989, in Asteroids II, ed. R. P. Binzel, T. Gehrels, \& M. S. Mathews (Tucson: University of Arizona Press), 524

Cohen, M., Walker, R. G., Carter, B., et al. 1999, AJ, 117, 1864

Delbó, M., \& Harris, A. W. 2002, Met. Planet. Science, 36, 1929

Fornasier, S., Belskaya, I., Fulchignoni, M., Barucci, M. A., \& Barbieri, C. 2006, A\&A, 449, L9

Gil-Hutton, R. 2006, A\&A, 464, 1127

Goldberg, D. E. 1989, Genetic algorithms in search, optimization and machine learning (Addison-Wesley, MA)
Hapke, B. 1993, Theory of reflectance and emittance spectroscopy (Cambridge University Press)

Hicks, M. D., Bauer, J. M., \& Tokunaga, A. T. 2004, IAU Circ., 8315

Holland, J. H. 1992, Adaptation in natural and artificial sustems (Cambridge, MA: MIT Press)

Kamei, A., \& Nakamura, M. 2002, Icarus, 156, 551

Käufl, H.-U., Sterzik, M. F., Siebenmorgen, R., et al. 2003, SPIE, 4841, 117

Küppers, M., Mottola, S., Lowry, S. C., et al. 2007, A\&A, 462, L13

Lazzarin, M., Marchi, S., Magrin, S., \& Barbieri, C. 2004, A\&A, 425, L25

Lagage, P. O., Pel, J. W., Authier, M., et al. 2004, The Messenger, 117, 12

Lagerros, J. S. 1996, A\&A, 315, 625

Lupishko, D. F., \& Belskaya, I. N. 1989, Icarus, 78, 395

Magri, C., Ostro, S. J., Rosema, K. D., et al. 1999, Icarus, 140, 379

Mueller, M., Harris, A. W., Bus, S. J., et al. 2006, A\&A, 447, 1153

Mahfoud, S. W. 1995, Ph.d. dissertation. Department of General Engeneering, University of Illinois at Urbana-Champaing

Nedelcu, A., Birlan, M., Vernazza, P., et al. 2007, A\&A, 470, 1157

Ostro, S. J., Campbell, D. B., \& Shapiro, I. I. 1985, Science, 229, 442

Piironen, J., Muinonen, K., Nousiainen, T., et al. 1998, Planet. Space Sci., 48, 937

Rivkin, A. S., Howell, E. S., Lebofsky, L. A., Clark, B. E., \& Britt, D. T. 2000, Icarus, 145,351

Spencer, J. R. 1990, Icarus, 83, 27

Tedesco, E. F., Noha, P. V., \& Price, S. D. 2004, NASA Planetary Data System, IRAS-A-FPA-3-RDR-IMPS-V6.0

Torppa, J., Kaasalainen, M., Michalowski, T., et al. 2003, Icarus, 164, 346

Weissman, P. R., Lowry, S. C., \& Choi, Y. 2007

[arXiv: astro-ph/0702339v1]

Zellner, B., \& Gradie, J. 1976, AJ, 81, 262 